\title{
Psychiatric comorbidity and medication use in autism: a community survey
}

\author{
AIMS AND METHOD \\ Individuals with autism often have \\ complex needs. In this paper, we \\ attempt to establish the prevalence \\ rates of other major psychiatric ill- \\ nesses in learning disabled adults \\ diagnosed with autism and analyse \\ the use of medication in these indivi- \\ duals. A total of 571 clients with \\ learning disability were screened for \\ autistic spectrum disorder, identi- \\ fying 164 individuals for study.
}

\author{
Psychiatric and medical diagnoses \\ were also reviewed.
RESULTS
Of the individuals with autism, 35\% had another comorbid psychiatric disorder. An overall prevalence rate of $10 \%$ for hypothyroidism was also identified and $35 \%$ of people with autism and no other psychiatric diagnosis were on neuroleptics.

\author{
CLINICAL IMPLICATIONS \\ Planning of services for autism \\ will have to take into account the \\ high comorbidity of autism and \\ other psychiatric conditions. \\ Individuals with autism will also \\ benefit from close monitoring of \\ mental state and the development \\ of hypothyroidism.
}

Autism as a concept has developed over the past 50 years since Kanner first described it in 1943 (Kanner, 1943). The concept of a classic triad of symptoms has developed into the present model of a continuum of related disorders, referred to as pervasive developmental disorders, with classic Kannerian autism lying at one extreme. It also appears that the prevalence of autism is increasing. Fombonne (1999), in his meta-analysis, showed an overall median rate for autism of 5.2/10 000 but studies over the past 10 years have shown a median rate of 7.2/10 000. It is not known if this increased rate is due to a true increase in incidence or to an increased awareness. A previous study by the authors showed a rate of 7.7/10 000 (Morgan et al, 2002).

The link between learning disability and autism was established in the early descriptions of the condition; more recent studies have quantified this relationship, suggesting that four out of five individuals with autism have a learning disability (Wing \& Gould, 1979). Individuals with autism can also be diagnosed with other psychiatric conditions: DeLong \& Nohria (1994) have suggested that autistic spectrum disorders may be related aetiologically to bipolar affective disorder, and Bolton et al (1998) suggest a possible link to affective disorders.

Research in individuals with both learning disabilities and autism are problematic because it is difficult to separate the deficits caused by autism from those due to a learning disability. The other consideration is that the presentation of psychiatric symptoms is transformed by the presence of autism and learning disabilities. Recognition of the impact of learning disabilities in changing the symptoms of psychiatric illness has led to the development of specific psychiatric diagnostic criteria for those with a learning disability (DC-LD; Royal College of Psychiatrists, 2001).
In view of the association between learning disabilities and autism, and autism and affective disorders, our aim was to study the psychiatric characteristics of adults with comorbid learning disabilities and autism.

To simplify discussion, the term 'autistic disorder' is used in this paper, which includes those with a diagnosis of atypical autism and childhood autism.

\section{Method}

The study population was identified in a two-stage method. Firstly, adults with learning disability attending any social, voluntary sector, private sector or National Health Service (NHS) service within an urban NHS Trust area (adult population of 230000 ) were screened for pervasive developmental disorders using the Pervasive Developmental Disorder Mental Retardation Scale (PDDMRS; Kraijer, 1997). The screening tool devised by Kraijer (1997) has 12 items and looks at behaviour in the past 2-6 months, as rated by a parent or carer. It is designed to be used in all levels of learning disability and from 2 to 55 years of age. Sensitivity is stated as $94 \%$ against the diagnosis of experienced psychological and medical experts, specificity is $93 \%$, the interrater reliability $(\kappa)$ is 0.83 and the scale was standardised on 1052 clients.

The PDD-MRS identified 153 positively tested individuals. All were found, on clinical examination by a learning disability consultant psychiatrist, to meet the ICD-10 (World Health Organization, 1992) criteria for autistic spectrum disorder. Of the 27 people identified as borderline with the PDD-MRS, 18 were clinically confirmed to have an autistic spectrum disorder by meeting the ICD-10 criteria.

Thus, 171 clients with autistic spectrum disorders were identified from 571 adults screened. Because the initial screening took 2 years, seven clients were lost to follow-up, leaving 164 clients. The 400 adults without a 
diagnosis of autism were used as a control group for prevalence rates of major psychiatric illness.

Additional data were collected from psychiatric case notes. As well as basic demographic data, information was collected on recorded medical and psychiatric comorbidity and current medication on a set day, the psychiatric diagnoses in the medical notes being based upon ICD-10 criteria. The level of learning disability was based on the information recorded in the clinical summary sheet contained in individual clinical notes.

\section{Results}

A total of 164 adults were identified with a diagnosis of autistic spectrum disorder. The overall female:male ratio

\section{Table 1. Level of learning disability in sample}

\begin{tabular}{lccc} 
& Mild & Moderate & Severe \\
\hline Psychiatric diagnosis & 13 & 18 & 37 \\
No psychiatric diagnosis & 7 & 33 & 56 \\
Total & 20 & 51 & 93 \\
& $(12 \%)$ & $(31 \%)$ & $(57 \%)$ \\
\hline
\end{tabular}

was 1:1.25, which is similar to other studies on the gender ratio of autism, although gender ratios for autism vary widely (Fombonne, 1999). Of the study group identified, $57 \%$ had severe, $31 \%$ had moderate and $12 \%$ had mild learning disability (see Table 1).

Most of the sample with autistic spectrum disorder did not have a definitive cause for their learning disability recorded, but of those whose diagnoses were known, 11 had Down's syndrome, two had tuberous sclerosis and one each had metachromatic leucodystrophy, fragile- $X$ syndrome, Cornelia de Lange syndrome and phenylketonuria.

The prevalence rates for psychiatric morbidity were: $41 \%$ within the whole sample; $65 \%$ in people with mild learning disability; $35 \%$ in those with moderate learning disability; and $40 \%$ in those with severe learning disability. People with severe learning disability accounted for $53 \%$ of all the psychiatric morbidity within the total sample (see Table 2).

Prevalence rates for the major psychiatric illnesses are shown in Table 3, which compares our results with the community studies of Lund (1985), Corbett (1979) and Cooper (1997). Using the screened individuals who did not have autism as a control group, significantly larger than expected lifetime prevalence rates of depression $(20 \%$ v. $2.75 \%)$ and bipolar affective disorder $(11 \%$ v.

\section{Table 2. Psychiatric diagnosis and levels of learning disability in autistic sample}

\begin{tabular}{lcccc} 
& \multicolumn{3}{c}{ Degree of learning disability } & Total individuals \\
\cline { 2 - 4 } Psychiatric diagnosis & Mild & Moderate & Severe & 18 \\
\hline $\begin{array}{l}\text { Bipolar disorder } \\
\text { Depression }\end{array}$ & 3 & 5 & 10 & 34 \\
Pica & 6 & 7 & 21 & 2 \\
Schizoaffective disorder & 1 & 1 & 2 & 2 \\
Schizophrenia & 3 & 5 & 4 & 8 \\
Tourette syndrome & 13 & 18 & 37 & 4 \\
Total & & & & 68 \\
\hline
\end{tabular}

Table 3. Psychiatric and medical diagnoses in autistic sample

\begin{tabular}{|c|c|c|c|c|c|c|}
\hline Diagnosis & Number (\%) & $95 \% \mathrm{Cl}$ & Corbett $^{1}(\%)$ & Lund $^{2}(\%)$ & Cooper $^{3}(\%)$ & Control $^{4}(\%)$ \\
\hline Depression & $34(20)^{*}$ & $15-27$ & 4 & 0.6 & 8 & 2.75 \\
\hline Bipolar affective disorder & $18(11)^{\star}$ & $7-17$ & 3.5 & 1 & 1.4 & 1.75 \\
\hline Schizophrenia & 8 (5) & $3-9$ & 6.5 & 1.3 & 3 & 5.25 \\
\hline Schizoaffective disorder & $2(1)$ & & & & & \\
\hline Tourette syndrome & $4 \quad(2)$ & & & & & \\
\hline Epilepsy & $56(34)$ & $27-41$ & & & & \\
\hline Hypothyroidism & $6(6)$ & $3-12$ & & & & \\
\hline Down's+hypothryoidism & $4 \quad(2)$ & & & & & \\
\hline Hypertension & $2(1)$ & & & & & \\
\hline Diabetes & $2(1)$ & & & & & \\
\hline
\end{tabular}

1. Corbett (1979), $n=402$

2. Lund (1985), $n=302$.

3. Cooper (1997) $n=73$.

4. Non-autistic clients, $n=400$

${ }^{*} P<0.02$ compared with each of the three community studies. 
Table 4. Neuroleptic use in individuals with autism

original papers

\begin{tabular}{lcc}
\hline Drug & All ${ }^{1}(n=164)$ & Autism only $(n=96)$ \\
\hline Chlorpromazine & 2 & 2 \\
Zuclopenthixol & 4 & 2 \\
Depot & 7 & 2 \\
Droperidol & 4 & 1 \\
Haloperidol & 19 & 7 \\
Olanzapine & 1 & 0 \\
Pericyazine & 3 & 3 \\
Pimozide & 1 & 0 \\
Promazine & 1 & 1 \\
Quetiapine & 1 & 1 \\
Resperidone & 19 & 5 \\
Sulpiride & 2 & 0 \\
Thioridizine & 21 & 10 \\
Trifluoperazine & 1 & 1 \\
Total & 87 & 35 (36\%) \\
\hline 1. Number of individuals with autism on each neuroleptic. & \\
2. Number of individuals on each neuroleptic with a sole diagnosis of autism and \\
learning disability.
\end{tabular}

$1.75 \%$ ) were found. Schizophrenia (5\% v. 5.25\%) did not show a significant difference, even when compared with the higher published population rates in people with learning disabilities. A rate of $2 \%$ for Tourette syndrome appeared high, but no comparison prevalence rates in learning disability were identified in a literature search. As would be expected in this sample, with a large number of people with severe and moderate learning disability, there was a high prevalence of epilepsy (34\%).

In the sample, $50 \%$ of the individuals were on at least one psychotropic medication and were on more than one. Psychotropic use is shown in Table 4. Of the 87 individuals on neuroleptics, 35 did not have an additional psychiatric diagnosis.

There was only one individual on antidepressants without a diagnosis of an affective disorder. In the subgroup with an affective disorder, citalopram was prescribed most frequently ( $8 / 21$ prescriptions of antidepressants) and the next most frequent were mirtazapine and fluoxetine, with three prescriptions each. Mood stabilisers in individuals without epilepsy were used only in individuals with recurrent depression or bipolar affective disorder. Carbamazepine was prescribed most frequently (10/18 prescriptions of mood stabilisers), seven individuals were on lithium, and one person was on both lithium and carbamazepine.

\section{Discussion}

Our population rate of autistic disorder was 7.7/10 000, and the median rate of autism for studies using strict criteria in the past 10 years (Fombonne, 1999) is also 7.7/ 10000 . However, the latter rate includes $20 \%$ of individuals with no learning disability. It is acknowledged that our sample would miss clients who live at home and accessed no social or medical services, and this may have biased our sample, but concurrence of the rates suggests a face validity for our sample method.

The male:female ratio of 1.25:1 approximates to most previous studies showing a male predominance for autism. Fombonne's meta-analysis showed a wide range of ratio from 0.6 to 2.7 in those with moderate and severe learning disabilities.

This study shows a high rate of psychiatric disorder among those with a diagnosis of autistic spectrum disorder, the largest rate being in the group with mild learning disabilities. A possible explanation is that this group have higher abilities that are proportionately more disabled by their autistic features and they are therefore more likely to develop psychiatric illnesses. Alternatively, psychopathology in those with more severe learning disabilities could be more likely to be attributed to the learning disability than to a psychiatric illness.

Comparison with published rates of psychiatric illness in people with learning disabilities (Cooper, 1997) shows that the group with autistic disorder had significantly increased rates of depression and bipolar affective disorder, the rate of schizophrenia appearing high but not reaching statistical significance. This seems to agree with the data of Lainhart \& Folstein (1994) and DeLong \& Nohria (1994), who have shown a higher incidence of affective disorders in the relatives of people with autism. This may suggest shared aetiological factors between autism and affective disorders, and warrants further investigation.

A surprising finding was the $10 \%$ prevalence of hypothyroidism, which is statistically significant compared with a general adult population rate of $1 \%$ or less (Tunbridge et al, 1977). On looking at this association, it was felt that there might have been other confounding factors. The rate was therefore recalculated by excluding individuals who had Down's syndrome, a history of affective disorders, or were on lithium or carbamazepine, all of which have been associated with hypothyroidism. The resulting prevalence rate of $6 \%$ is still significantly high and therefore, it may be advisable to screen all clients with autistic disorder for hypothyroidism, or at least be on the alert for its presence, especially as it is also associated with depression.

As far as medication is concerned, one can only use the information gained empirically because there are no outcome data. The medication recorded was the client's regular current medication, which would suggest that the client had been stabilised on the medication and it had been perceived to be of benefit. The data were collected prior to the change in the licence of thioridazine.

Overall, $52 \%$ of autistic individuals were on psychotropics and $40 \%$ of those had autistic disorder as the sole psychiatric diagnosis. Clarke et al (1990) found that 36\% of learning disabled individuals who did not have a diagnosis of psychiatric illness received psychotropic medication, suggesting that the main indication for the use of psychotropic medication in these people was behavioural disorder. It is therefore assumed in our study that the medication was used to reduce behaviour associated with autism, such as stereotypy and aggression. Thioridazine, 
haloperidol and risperidone were used most frequently. Posey \& McDougle (2000) reviewed drug treatment in pervasive developmental disorders and suggested some reported benefits for the use of haloperidol and risperidone in autism, thioridazine previously being licensed for problematic behaviour. Randomised controlled trials for the drug treatment of autism are few, but McDougle et al (1998) found risperidone to be effective in controlling aggression in a double-blind, randomised controlled trial of subjects with autism, confirming the drug's usefulness. Because neuroleptic medications appear to be used extensively for the treatment of behavioural difficulties with autism, there is clearly a need to study this further.

Carbamazepine was used more frequently than lithium as a mood stabiliser. This may reflect the difficulties of monitoring serum lithium in people with learning disabilities in general, and with autistic disorders in particular

\section{Conclusion}

This study shows a high lifetime prevalence rate of psychiatric disorders and of hypothyroidism in learning disabled adults with a diagnosis of autistic spectrum disorder. The clinical implications are that we need to be alert to psychiatric comorbidity and check for hypothyroidism regularly.

This has general implications for resources and also individual treatment. Such individuals will have very complex needs and will require specialised treatment packages to enable them to develop their full potential.

\section{Acknowledgement}

The authors would like to thank Dr Mlele for facilitating this project.

\section{References}

BOLTON, P. F., PICKLES, A. \& MURPHY, LUND, J. (1985) The prevalence of M. (1998) Autism, affective and other psychiatric disorders: patterns of familiar aggregation. Psychological Medicine, 28, 385-395.

CLARKE, D. J., KELLY, S. \& THINN, K. (1990) Psychotropic drugs and mental retardation. 1. Disabilities and the prescription of drugs for behaviour and epilepsy in three residential settings. Journal of Mental Deficiency Research, 34, 385-395

COOPER, S. A. (1997) Epidemiology of psychiatric disorders in elderly compared with younger adults with learning disabilities. British Journal of Psychiatry, 170, 375-380.

CORBETT, J. A. (1979) Psychiatric morbidity and mental retardation. In Psychiatric Illness and Mental Handicap (eds F. E. James \& R. P. Snaith), pp.1126. Ashford, Kent: Headley Brothers.

DELONG, R. \& NOHRIA, C. (1994)

Psychiatric family history and neurological disease in autistic spectrum disorders. Developmental

Medicine and Child Neurology, 36, 441-448.

FOMBONNE, E. (1999) The epidemiology of autism: a review. Psychological Medicine, 29, 769-786.

KANNER, L. (1943) Autistic disturbances of affective contact. Nervous Child, 2, 217-250.

KRAIJER, D.W. (1997) Autism and Autistic-like Conditions in Mental Retardation. Lisse: Swets \& Zeitlinger.

LAINHART, J. E. \& FOLSTEIN, S. E. (1994) Affective disorders in people with autism: a review of published cases. Journal of Autism and Developmental Disorders, 24, 587-601. psychiatric morbidity in mentally retarded adults. Acta Psychiatrica Scandinavica, 72, 563-570.

MCDOUGLE, C. J., HOLMES, J. P. CARLSON, J. C., et al (1998) A double blind, placebo-controlled study of risperidone in adults with autistic disorder and other pervasive developmental disorders. Archives of General Psychiatry, 55, 633-641.

MORGAN, C. N., ROY, M., NASR, A., et al (2002) A community study establishing the prevalence rate of autistic disorder in adults with learning disability. Psychiatric Bulletin, 26, 127-129.

POSEY, D. J. \& MCDOUGLE, C. J. (2000) The pharmacology of target symptoms associated with autistic disorder and other pervasive developmental disorders. Harvard Review of Psychiatry, July/Aug, 45-63. ROYAL COLLEGE OF PSYCHIATRISTS (2001) DC-LD: Diagnostic Criteria for Psychiatric Disorders for Use with Adults with Learning Disabilities/ Mental Retardation. London: Gaskell.

TUNBRIDGE, W. M., EVERED, D. C \& HALL, R. (1977) The spectrum of thyroid disease in a community: theWhickham survey. Clinical Endocrinology, 7 481-493.

WING, L. \& GOULD, J. (1979) Severe impairments of social interaction and associated abnormalities in children: epidemiology and classification. Journal of Autism and Childhood Schizophrenia, 9, 11-29.

WORLD HEALTH ORGANIZATION (1992) Tenth Revision of the International Classification of Diseases and Related Health Problems (ICD-10). Geneva: WHO.

*Caryl N. Morgan Consultant in Psychiatry of Learning Disability, Brian Olive Centre, Brooklands Hospital, Coleshill Road, Marston Green, Birmingham B37 7HL, Meera Roy Consultant in Psychiatry of Learning Disability, Birmingham Specialist Community Health NHS Trust, Birmingham,

Patrick Chance Staff Grade Psychiatrist, Ladywood HomeTreatment Team, Birmingham original

papers 